\title{
Predictive Function Control of the Single-Link Manipulator with Flexible Joint
}

\author{
Zhihuan Zhang and Chao $\mathrm{Hu}$ \\ Ningbo Institute of Technology, Zhejiang University
}

China

\section{Introduction}

Flexible-link robotic manipulators have many advantages with respect to conventional rigid robots. They are built by lighter, cheaper materials, which improve the payload to arm weight ratio, thus resulting in an increase of the speed with lower energy consumption. Moreover, due to the reduced inertia and compliant structure, these lightweight arms can be operated more safely and are more applicable for the delicate assembly tasks and interaction with fragile objects, including human beings.

The control for robot manipulators is to determine the time history of joint inputs to cause the end-effector to execute a commanded motion. There are many control techniques and methodologies that can be applied to the control of the manipulators. The specific control method and its implementation ways can have a significant impact on the performance of the manipulator and consequently on the range of its possible applications. In addition, the mechanical design of the manipulator itself will influence the type of control scheme needed. However, in order to improve the control performance, more sophisticated approaches should be found.

The control for flexible joint system has attracted a considerable amount of attention during the past few years. There are PD, inverse dynamics, and the force control approach for the feedback control strategies of flexible joint manipulator. (1989, MARK W. SPONG), an integral manifold approach to the feedback control of flexible joint robots (1987, MARK W. SPONG, KHASHAYAR KHORASANI, and PETAR V. KOKOTOVIC), and the nonlinear feedback control of flexible joint manipulators: a single link case study, (1990, K. KHORASANI). The basic idea of feedback linearization is to construct a nonlinear control law as a so-called inner loop control which, in the ideal case, exactly linearizes the nonlinear system after a suitable state space change of coordinates. The designer can design a second stage or outer loop control in the new coordinates to satisfy the traditional control design specifications such as tracking, disturbance rejection, and so forth. Since the feedback linearization of flexible joint manipulator is a fourth order integrator system, so we proposed a three stage design method, the first is nonlinear feedback to get integrator system, the second is pole placement to get expect performance, and the third is to use PFC to reject disturbance and uncertainty, since they can not be exactly cancelled by nonlinear feedback, coupling effects of the joint flexibility. More accurate description of robot dynamics may include fast actuator dynamics and joint-link flexibility, and so on. 


\section{Equations of motion}

Consider the single-link arm shown in Figure 1 consisting of a flexible joint.

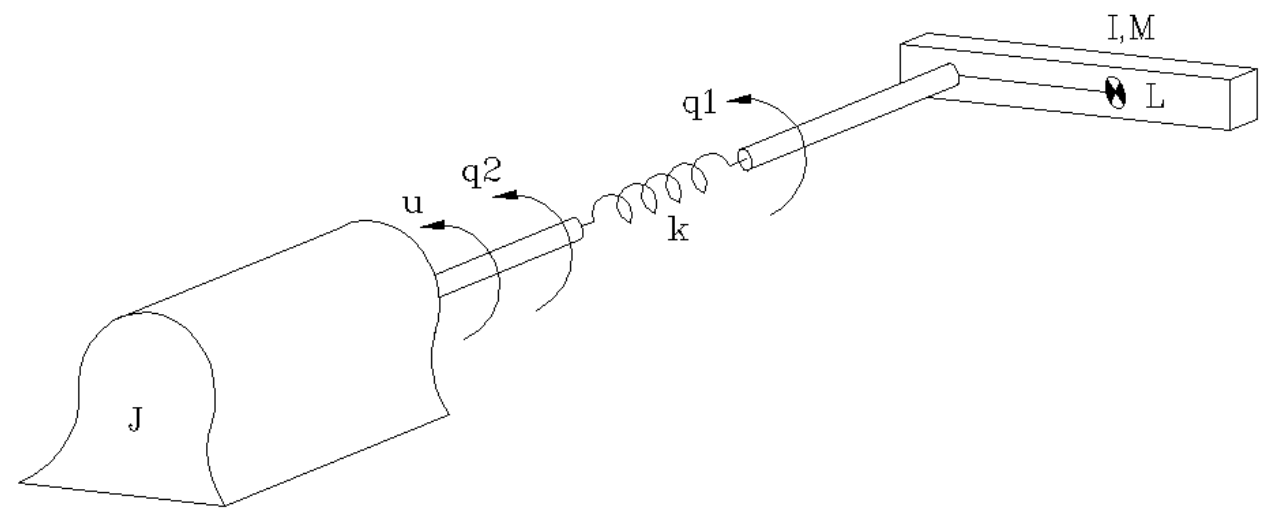

Fig. 1. Single-link robot with joint flexibility

The kinetic energy of the manipulator is a quadratic function of the vector $\dot{q}$

$$
K=\frac{1}{2} \dot{q}^{T} D(q) \dot{q}=\frac{1}{2} \sum_{i, j}^{n} d_{i j}(q) \dot{q}_{i} \dot{q}_{j}
$$

where the $n \times n$ inertia matrix $D(q)$ is symmetric and positive definite for each $q \in \mathfrak{R}^{n}$.

The potential energy $V=V(q)$ is independent of $\dot{q}$. We have remarked that robotic manipulator satisfies this condition.

The Euler-Lagrange equations for such a system can be derived as follows. Since

$$
L=K-V=\frac{1}{2} \sum_{i, j}^{n} d_{i j}(q) \dot{q}_{i} \dot{q}_{j}-V(q)
$$

we have

$$
\frac{\partial L}{\partial \dot{q}_{k}}=\sum_{j} d_{k j}(q) \dot{q}_{j}
$$

and

$$
\frac{d}{d t} \frac{\partial L}{\partial \dot{q}_{k}}=\sum_{j} d_{k j}(q) \ddot{q}_{j}+\sum_{j} \frac{d}{d t} d_{k j}(q) \dot{q}_{j}=\sum_{j} d_{k j}(q) \ddot{q}_{j}+\sum_{i, j} \frac{\partial d_{k j}}{\partial q_{i}} \dot{q}_{i} \dot{q}_{j}
$$

Also 


$$
\frac{\partial L}{\partial q_{k}}=\frac{1}{2} \sum_{i, j} \frac{\partial d_{i j}}{\partial q_{k}} \dot{q}_{i} \dot{q}_{j}-\frac{\partial V}{\partial q_{k}}
$$

Thus the Euler-Lagrange equations can be written as

$$
\sum_{j} d_{k j}(q) \ddot{q}_{j}+\sum_{i, j}\left\{\frac{\partial d_{k j}}{\partial q_{i}}-\frac{1}{2} \frac{\partial d_{i j}}{\partial q_{k}}\right\} \dot{q}_{i} \dot{q}_{j}-\frac{\partial V}{\partial q_{k}}=\tau_{k}, k=1, \cdots, n
$$

By interchanging the order of summation and taking advantage of symmetry, we can show that

$$
\sum_{i, j}\left\{\frac{\partial d_{k j}}{\partial q_{i}}\right\} \dot{q}_{i} \dot{q}_{j}=\frac{1}{2} \sum_{i, j}\left\{\frac{\partial d_{k j}}{\partial q_{i}}+\frac{\partial d_{k i}}{\partial q_{j}}\right\} \dot{q}_{i} \dot{q}_{j}
$$

Hence

$$
\sum_{i, j}\left\{\frac{\partial d_{k j}}{\partial q_{i}}-\frac{1}{2} \frac{\partial d_{i j}}{\partial q_{k}}\right\} \dot{q}_{i} \dot{q}_{j}=\sum_{i, j} \frac{1}{2}\left\{\frac{\partial d_{k j}}{\partial q_{i}}+\frac{\partial d_{k i}}{\partial q_{j}}-\frac{\partial d_{i j}}{\partial q_{k}}\right\} \dot{q}_{i} \dot{q}_{j}
$$

The term

$$
c_{i j k}=\frac{1}{2}\left\{\frac{\partial d_{k j}}{\partial q_{i}}+\frac{\partial d_{k i}}{\partial q_{j}}-\frac{\partial d_{i j}}{\partial q_{k}}\right\}
$$

are known as Christoffel symbols. Note that, for a fixed $\mathrm{k}$, we have $c_{i j k}=c_{j i k}$, which reduces the effort involved in computing these symbols by a factor of about one half. Finally, if we define

$$
\varphi_{k}=\frac{\partial V}{\partial q_{k}}
$$

then the Euler-Lagrange equations can be written as

$$
\sum_{j} d_{k j}(q) \ddot{q}_{j}+\sum_{i, j} c_{i j k}(q) \dot{q}_{i} \dot{q}_{j}+\varphi_{k}(q)=\tau_{k}, k=1, \cdots, n
$$

In the above equation, there are three types of terms. The first involve the second derivative of the generalized coordinates. The second are quadratic terms in the first derivatives of $q$, where the coefficients may depend on $\mathrm{q}$. These are further classified into two types. Terms involving a product of the type $\dot{q}_{i}^{2}$ are called centrifugal, while those involving a product of the type $\dot{q}_{i} \dot{q}_{j}$ where $i \neq j$ are called Coriolis terms. The third type of terms are those involving only $\mathrm{q}$ but not its derivatives. Clearly the latter arise from differentiating the potential energy. It is common to write (6) in matrix form as

$$
D(q) \ddot{q}+C(q, \dot{q}) \dot{q}+g(q)=\tau
$$


where the $\mathrm{k}$, j-th element of the matrix $C(q, \dot{q})$ is defined as

$$
c_{k j}=\sum_{i=1}^{n} c_{i j k}(q) \dot{q}_{i}=\sum_{i=1}^{n} \frac{1}{2}\left\{\frac{\partial d_{k j}}{\partial q_{i}}+\frac{\partial d_{k i}}{\partial q_{j}}-\frac{\partial d_{i j}}{\partial q_{k}}\right\} \dot{q}_{i}
$$

\section{Feedback linearization design for inner loop}

We first derive a model similar to (6) to represent the dynamics of a single link robot with joint flexibility. For simplicity, ignoring damping of the equations of motion, system is given by

$$
\begin{gathered}
D\left(q_{1}\right) \ddot{q}_{1}+h\left(q_{1}, \dot{q}_{1}\right) \dot{q}_{1}+K\left(q_{1}-q_{2}\right)=0 \\
J \ddot{q}_{2}-K\left(q_{1}-q_{2}\right)=u
\end{gathered}
$$

In state space, which is now $\mathfrak{R}^{4 n}$, we define state variables in block form

$$
\begin{array}{ll}
x_{1}=q_{1} & x_{2}=\dot{q}_{1} \\
x_{3}=q_{2} & x_{4}=\dot{q}_{2}
\end{array}
$$

Then from (8)-(9) we have

$$
\begin{gathered}
\dot{x}_{1}=x_{2} \\
\dot{x}_{2}=-D\left(x_{1}\right)^{-1}\left\{h\left(x_{1}, x_{2}\right)+K\left(x_{1}-x_{3}\right)\right\} \\
\dot{x}_{3}=x_{4} \\
\dot{x}_{4}=J^{-1} K\left(x_{1}-x_{3}\right)+J^{-1} u
\end{gathered}
$$

This system is then of the form

$$
\dot{x}=f(x)+G(x) u
$$

For a single-input nonlinear system, $f(x)$ and $g(x)$ are smooth vector fields on $\mathfrak{R}^{n}$, $f(0)=0$, and $u \in \mathfrak{R}$, is said to be feedback linearizable if there exists a region $\mathrm{U}$ in $\mathfrak{R}^{n}$ containing the origin, a diffeomorphism $\mathrm{T}: U \rightarrow \mathfrak{R}^{n}$, and nonlinear feedback

$$
u=\alpha(x)+\beta(x) v
$$

with $\beta(x) \neq 0$ on $\mathrm{U}$, such that the transformed variables

$$
y=T(x)
$$

satisfy the system of equations

$$
\dot{y}=A y+b v
$$

where 


$$
A=\left[\begin{array}{rrrrrr}
0 & 1 & 0 & & & 0 \\
0 & 0 & 1 & & & . \\
\cdot & \cdot & . & . & & \cdot \\
\cdot & . & . & & . & . \\
. & . & . & & & 1 \\
0 & 0 & . & . & 0 & 0
\end{array}\right] \quad b=\left[\begin{array}{c}
0 \\
0 \\
\cdot \\
\cdot \\
\cdot \\
1
\end{array}\right]
$$

In the single-link case we see that the appropriate state variables with which to define the system so that it could be linearized by nonlinear feedback on the link position, velocity, acceleration, and jerk. Following the single-input case, then, we can apply same action on the multi-link case and derive a feedback linearizing transformation blockwise as follows,

$$
\begin{gathered}
y_{1}=T_{1}(x)=x_{1} \\
y_{2}=T_{2}(x)=\dot{y}_{1}=x_{2} \\
y_{3}=T_{3}(x)=\dot{y}_{2}=\dot{x}_{2}=-D\left(x_{1}\right)^{-1}\left\{h\left(x_{1}, x_{2}\right)+K\left(x_{1}-x_{3}\right)\right\} \\
y_{4}=T_{4}(x)=\dot{y}_{3}=-\frac{d}{d t}\left[D\left(x_{1}\right)^{-1}\right]\left\{h\left(x_{1}, x_{2}\right)+K\left(x_{1}-x_{3}\right)\right\}-D\left(x_{1}\right)^{-1}\left\{\frac{\partial h}{\partial x_{1}} x_{2}\right. \\
\left.+\frac{\partial h}{\partial x_{2}}\left[-D\left(x_{1}\right)^{-1}\left(h\left(x_{1}, x_{2}\right)+K\left(x_{1}-x_{3}\right)\right)\right]+K\left(x_{2}-x_{4}\right)\right\}=a_{4}\left(x_{1}, x_{2}, x_{3}\right)+D\left(x_{1}\right)^{-1} K x_{4}
\end{gathered}
$$

where for simplicity we define the function $a_{4}$ to be that in the definition of $y_{4}$ except the last term, which is $D^{-1} K x_{4}$. Note that $x_{4}$ appears only in this last term so that $a_{4}$ depends only on $x_{1}, x_{2}, x_{3}$.

As in the single-link case, the above mapping is a global diffeomorphism. Its inverse can be found by

$$
\begin{gathered}
x_{1}=y_{1} \\
x_{2}=y_{2} \\
x_{3}=y_{1}+K^{-1}\left(D\left(y_{1}\right) y_{3}+h\left(y_{1}, y_{2}\right)\right) \\
x_{4}=K^{-1} D\left(y_{1}\right)\left(y_{4}-a_{4}\left(y_{1}, y_{2}, y_{3}\right)\right)
\end{gathered}
$$

The linearizing control law can now be found from the condition

$$
y_{4}=v
$$

where $v$ is a new control input. Computing $\dot{y}_{4}$ from (22) and suppressing function arguments for brevity yields 


$$
\begin{aligned}
& v=\frac{\partial a_{4}}{\partial x_{1}} x_{2}-\frac{\partial a_{4}}{\partial x_{2}} D^{-1}\left(h+K\left(x_{1}-x_{3}\right)\right)+\frac{\partial a_{4}}{\partial x_{3}} x_{4}+\frac{d}{d t}\left[D^{-1}\right] K x_{4}+D^{-1} K\left(J^{-1} K\left(x_{1}-x_{3}\right)+J^{-1} u\right) \\
& =a(x)+b(x) u
\end{aligned}
$$

where

$$
\begin{gathered}
a(x)=: \frac{\partial a_{4}}{\partial x_{1}} x_{2}-\frac{\partial a_{4}}{\partial x_{2}} D^{-1}\left(h+K\left(x_{1}-x_{3}\right)\right)+\frac{\partial a_{4}}{\partial x_{3}} x_{4}+\frac{d}{d t}\left[D^{-1}\right] K x_{4}+D^{-1} K J^{-1} K\left(x_{1}-x_{3}\right) \\
b(x)=D^{-1}(x) K J^{-1} u
\end{gathered}
$$

Solving the above expression for $\mathrm{u}$ yields

$$
\begin{aligned}
u & =b(x)^{-1}(v-a(x)) \\
& =: \alpha(x)+\beta(x) v
\end{aligned}
$$

where $\beta(x)=J K^{-1} D(x)$ and $\alpha(x)=-b(x)^{-1} a(x)$

With the nonlinear change of coordinates (19)-(22) and nonlinear feedback (32) the transformed system has the linear block form

$$
\begin{gathered}
\dot{y}=\left[\begin{array}{llll}
0 & I & 0 & 0 \\
0 & 0 & I & 0 \\
0 & 0 & 0 & I \\
0 & 0 & 0 & 0
\end{array}\right] y+\left[\begin{array}{l}
0 \\
0 \\
0 \\
I
\end{array}\right] v \\
=: A y+b v
\end{gathered}
$$

where $I=n \times n$ identity matrix, $0=n \times n$ zero matrix, $y^{T}=\left(y_{1}^{T}, y_{2}^{T}, y_{3}^{T}, y_{4}^{T}\right) \in \mathfrak{R}^{4 n}$, and $v \in \mathfrak{R}^{n}$. The system (33) represents a set of $\mathrm{n}$ decoupled quadruple integrators.

\section{Outer loop design based on predictive function control}

\section{1 why use predictive function control}

The technique of feedback linearization is important due to it leads to a control design methodology for nonlinear systems. In the context of control theory, however, one should be highly suspicious of techniques that rely on exact mathematical cancellation of terms, linear or nonlinear, from the equations defining the system.

In this section, we investigate the effect of parameter uncertainty, computational error, model simplification, and etc. We show that the most important property of feedback linearizable systems is not necessarily that the nonlinearities can be exactly cancelled by nonlinear feedback, but rather that, once an appropriate coordinate system is found in which the system can be linearized, the nonlinearities are in the range space of the input. This property is highly significant and is exploited by the predictive function control techniques to guarantee performance in the realistic case that the nonlinearities in the system are not known exactly. 
Consider a single-input feedback linearizable system. After the appropriate coordinate transformation, the system can be written in the ideal case as

$$
\begin{aligned}
\dot{y}_{1} & =y_{2} \\
& \vdots \\
& \vdots \\
\dot{y}_{n} & =v=\beta^{-1}(x)[u-\alpha(x)]
\end{aligned}
$$

provided that $\mathrm{u}$ is given by (16) in order to cancel the nonlinear terms $\alpha(x)$ and $\beta(x)$.

In practice such exact cancellation is not achievable and it is more realistic to suppose that the control law $u$ in (16) is of the form

$$
u=\hat{\alpha}(x)+\hat{\beta}(x) v
$$

where $\hat{\alpha}(x), \hat{\beta}(x)$ represent the computed versions of $\alpha(x), \beta(x)$, respectively. These functions may differ from the true $\alpha(x), \beta(x)$ for several reasons. Because the inner loop control $\mathrm{u}$ is implemented digitally, there will be an error due to computational round-off and delay. Also, since the terms $\alpha(x), \beta(x)$ are functions of the system parameters such as masses, and moments of inertia, any uncertainty in knowledge of these parameters will be in reflected in $\hat{\alpha}(x), \hat{\beta}(x)$. In addition, one may choose intentionally to simplify the control $\mathrm{u}$ by dropping various terms in the equations in order to facilitate on-line computation. If we now substitute the control law (35) into (34) we obtain

$$
\begin{aligned}
& \dot{y}_{1}=y_{2} \\
& \quad \vdots \\
& \quad \vdots \\
& \dot{y}_{n}=v=\beta^{-1}(x)[\hat{\alpha}(x)+\hat{\beta}(x) v-\alpha(x)] \\
& =v+\eta\left(y_{1}, \cdots, y_{n}, v\right)
\end{aligned}
$$

where the uncertainty $\eta$ is given as

$$
\eta\left(y_{1}, \cdots, y_{n}, v\right)=\left.\left\{\left(\beta^{-1} \hat{\beta}-1\right) v+\beta^{-1}(\hat{\alpha}-\alpha)\right\}\right|_{y=T^{-1}(X)}
$$

The system (36) can be written in matrix form as

$$
\dot{y}=A y+b\{v+\eta(y, v)\}
$$

where $\mathrm{A}$ and $\mathrm{b}$ are given by (18). For multi-input case, similar to (33), and if $v \in \mathfrak{R}^{m}$, and $\eta: \mathfrak{R}^{n} \times \mathfrak{R}^{m} \rightarrow \mathfrak{R}^{m}$. Note that the system (38) is still nonlinear whenever $\eta \neq 0$. The practical implication of this is solved by the outer loop predictive function control (PFC).

The system (38) can be represented by the block diagram of Figure 2. The application of the nonlinear inner loop control law results in a system which is "approximately linear". A common approach is to decompose the control input $v$ in (38) into two parts, the first to 
stabilize the 'nominal linear system' represented by (38) with $\eta=0$. In this case $v$ can be taken as a linear state feedback control law designed to stabilize the nominal system and/or for tracking a desired trajectory. A second stage control $\Delta v$ is then designed for robustness, that is, to guarantee the performance of the nominal design in the case that $\eta \neq 0$. Thus the form of the control law is

$$
\begin{gathered}
u=\hat{\alpha}(x)+\hat{\beta}(x) v \\
v=-K y+\Delta v \\
\Delta v=\operatorname{PFC}\left(y_{r}\right)
\end{gathered}
$$

where Ky is a linear feedback designed to place the eigenvalues of $\mathrm{A}$ in a desired location, $\Delta v$ represents an additional feedback loop to maintain the nominal performance despite the presence of the nonlinear term $\eta \cdot y_{r}$ is a reference input, which can be chosen as a signal for tracking a desired trajectory.

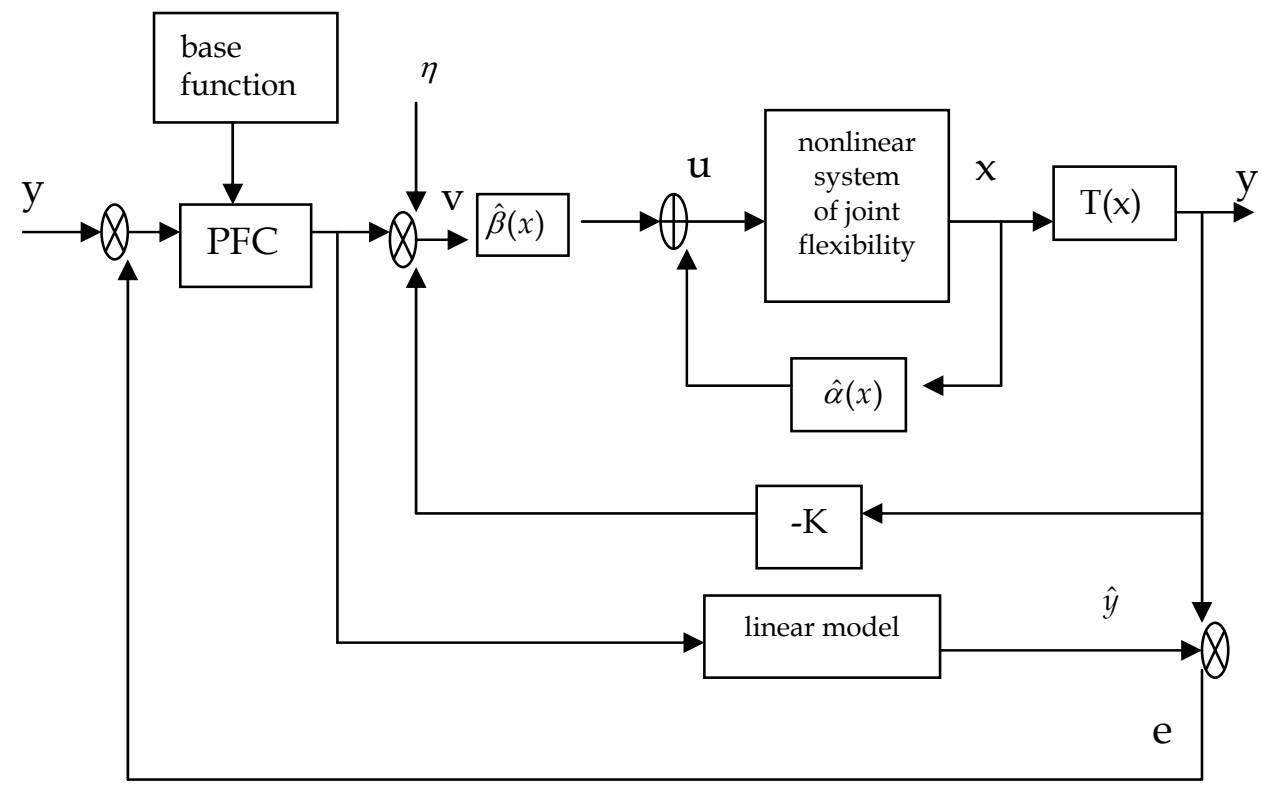

Fig. 2. block diagram for PFC outer loop design

\subsection{Predictive function control}

All MPC strategies use the same basic approach i.e., prediction of the future plant outputs, and calculation of the manipulated variable for an optimal control. Most MPC strategies are based on the following principles:

\section{Use of an internal model}

Its formulation is not restricted to a particular form, and the internal model can be linear, nonlinear, state space form, transfer function form, first principles, black-box etc. In PFC, 
only independent models where the model output is computed only with the present and past inputs of the process models are used.

\section{Specification of a reference trajectory}

Usually an exponential.

\section{Determination of the control law}

The control law is derived from the minimization of the error between the predicted output and the reference with the projection of the Manipulated Variable (MV) on a basis of functions.

Although based on these principles the PFC algorithm may be of several levels of complexity depending on the order and form of the internal model, the order of the basis function used to decompose the MV and the reference trajectory used.

\subsection{First order PFC}

Although it is unrealistic to represent industrial systems by a first order system, as most of them are in a higher order, some well behaved ones may be estimated by a first order. The estimation will not be perfect at each sample time, however, the robustness of the PFC will help to maintain a decent control.

If the system can be modelled by a first order plus pure time delay system, then the following steps in the development of the control law are taken.

\section{Model formulation}

In order to implement a basic first order PFC, a typical first order transfer function equation (42) is used.

$$
y_{M}(s)=\frac{K_{M}}{T_{M} S+1} u(s)
$$

Note that the time delay is not considered in the internal model formulation and in this case $K_{M}$ is equal to one. The discrete time formulation of the model zero-order hold equivalent is then obtained in (43).

$$
y_{M}(k)=\alpha y_{M}(k-1)+K_{M}(1-\alpha) u(k-1)
$$

where $\alpha=\exp \left(-\frac{T_{S}}{T_{M}}\right)$. If the manipulated variable is structured as a step basis function:

$$
\begin{gathered}
y_{L}(k+H)=\alpha^{H} y_{M}(k) \\
y_{F}(k+H)=K_{M}\left(1-\alpha^{H}\right) u(k)
\end{gathered}
$$

Where, $y_{L}$ and $y_{F}$ are respectively, the free (autoregressive) and the forced response of $y_{M}$.

\section{Reference trajectory formulation}

If $y_{R}$ is the expression of the reference trajectory, then at the coincidence point $\mathrm{H}$ : 


$$
C(k+H)-y_{R}(k+H)=\lambda^{H}\left(C(k)-y_{P}(k)\right)
$$

thus:

$$
y_{R}(k+H)=C(k)-\lambda^{H}\left(C(k)-y_{P}(k)\right)
$$

\section{Predicted process output}

The predicted process output is given by the model response, plus a term given the error between the same model output and the process output:

$$
\hat{y}_{P}(k+H)=y_{M}(k+H)+\left(y_{P}(k)-y_{M}(k)\right)
$$

where $y_{M}(k+H)=y_{L}(k+H)+y_{F}(k+H)=\alpha^{H} y_{M}(k)+K_{M}\left(1-\alpha^{H}\right) u(k)$.

\section{Computation of the control law}

At the coincidence point $\mathrm{H}$ :

$$
y_{R}(k+H)=\hat{y}_{P}(k+H)
$$

Combining (44), (45), (47) and (48) yields

$$
C(k)-\lambda^{H}\left(C(k)-y_{P}(k)\right)-y_{P}(k)=y_{M}(k+H)-y_{M}(k)
$$

Replacing $y_{M}(k+H)$ by its equivalent in equations (44) and (45) we obtain:

$$
C(k)\left(1-\lambda^{H}\right)-y_{P}(k)\left(1-\lambda^{H}\right)+y_{M}(k)\left(1-\alpha^{H}\right)=K_{M}\left(1-\alpha^{H}\right) u(k)
$$

Solving for $\mathrm{u}(\mathrm{k})$ the final result is the control law given in (52).

$$
u(k)=\frac{\left(C(k)-y_{P}(k)\right)\left(1-\lambda^{H}\right)}{K_{M}\left(1-\alpha^{H}\right)}+\frac{y_{M}(k)}{K_{M}}
$$

\subsection{Case of a process with a pure time delay}

In the linear case, a process with a pure time delay can be expressed in terms of a delay-free part, plus a delay added at the output, as in Fig. 3.

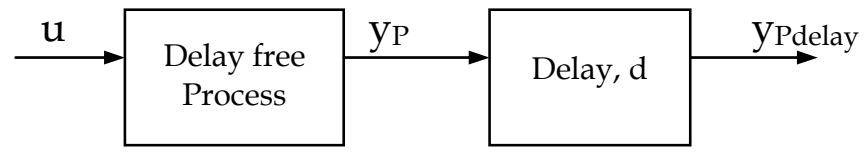

Fig. 3. Process with time delay

The value $y_{\text {Pdelay }}$ at time $\mathrm{k}$ is measured, but not $y_{P}$. In order to take into account the delay in a control law formulation, prior knowledge of the delay value $\mathrm{d}$ is needed. $y_{P}$ can be estimated as: 


$$
y_{P}(k)=y_{\text {Pdelay }}(k)+y_{M}(k)-y_{M}(k-d)
$$

\subsection{Tuning in PFC}

According to the three principles of PFC, tuning is a function of the order of the basis constructing the MV, the reference trajectory, the control horizon and the CLRT value.

The influence of the PFC parameters is given in Table 1, where the influence of various PFC parameters is on precision (Steady State Resp.), transient response and robustness are graded between 0 (indicating minimum influence) and 1 (indicating maximum influence).

\begin{tabular}{lccc}
\hline & SS Resp. & Transient Resp. & Robustness \\
\hline Basis function & 1 & 0 & 0 \\
Reference trajectory & 0 & 1 & $1 / 2$ \\
Coincidence horizon & 0 & $1 / 2$ & 1 \\
\hline
\end{tabular}

Table 1. Effect of PFC parameters in tuning

In most cases, an exponential reference trajectory is chosen along with a single coincidence horizon point $(\mathrm{H}=1)$ and a zero order basis function (Richalet, 1993). Considering the known Open Loop Response Time of the system (OLRT), one can choose the CLRT value given by the ratio OLRT/CLRT. This ratio then becomes the major tuning parameter shaping the system output and MV, dictating how much overshoot occurs and ensuring stability, on the condition that the internal model is accurate enough. For slow processes, e.g., heat exchange systems, a ratio of 4 or 5 is found most suitable, and ensures a stable PFC.

\section{Simulation}

Consider the single link manipulator with flexible joint shown in Figure 1. Choosing $q_{1}$ and $q_{2}$ as generalized coordinates, the kinetic energy is

$$
K=\frac{1}{2} I \dot{q}_{1}^{2}+\frac{1}{2} J \dot{q}_{2}^{2}
$$

The potential energy is

$$
V=\operatorname{MgL}\left(1-\cos q_{1}\right)+\frac{1}{2} k\left(q_{1}-q_{2}\right)^{2}
$$

The Lagrangian is

$$
L=K-V=\frac{1}{2} I \dot{q}_{1}^{2}+\frac{1}{2} J \dot{q}_{2}^{2}-M g L\left(1-\cos q_{1}\right)-\frac{1}{2} k\left(q_{1}-q_{2}\right)^{2}
$$

Therefore we compute 


$$
\begin{gathered}
\frac{\partial L}{\partial \dot{q}_{1}}=I \dot{q}_{1} \quad \frac{\partial L}{\partial \dot{q}_{2}}=J \dot{q}_{2} \\
\frac{d}{d t} \frac{\partial L}{\partial \dot{q}_{1}}=I \ddot{q}_{1} \quad \frac{d}{d t} \frac{\partial L}{\partial \dot{q}_{2}}=J \ddot{q}_{2} \\
\frac{\partial L}{\partial q_{1}}=-\operatorname{Mg} L \sin \left(q_{1}\right)-k\left(q_{1}-q_{2}\right) \quad \frac{\partial L}{\partial q_{2}}=k\left(q_{1}-q_{2}\right)
\end{gathered}
$$

Therefore the equations of motion, ignoring damping, are given by

$$
\begin{gathered}
I \ddot{q}_{1}+\operatorname{MgL} \sin \left(q_{1}\right)+k\left(q_{1}-q_{2}\right)=0 \\
J \ddot{q}_{2}-k\left(q_{1}-q_{2}\right)=u
\end{gathered}
$$

Note that since the nonlinearity enters into the first equation the control $u$ cannot simply be chosen to cancel it as in the case of the rigid manipulator equations. In other words, there is no obvious analogue of the inverse dynamics control for the system in this form.

In state space we set

$$
\begin{array}{ll}
x_{1}=q_{1} & x_{2}=\dot{q}_{1} \\
x_{3}=q_{2} & x_{4}=\dot{q}_{2}
\end{array}
$$

and write the system (60)- (61) as

$$
\begin{gathered}
\dot{x}_{1}=x_{2} \\
\dot{x}_{2}=-\frac{M g L}{I} \sin \left(x_{1}\right)-\frac{k}{I}\left(x_{1}-x_{3}\right) \\
\dot{x}_{3}=x_{4} \\
\dot{x}_{4}=\frac{k}{J}\left(x_{1}-x_{3}\right)+\frac{1}{J} u
\end{gathered}
$$

The system is thus of the form (15) with

$$
f(x)=\left[\begin{array}{c}
x_{2} \\
-\frac{M g L}{I} \sin \left(x_{1}\right)-\frac{k}{I}\left(x_{1}-x_{3}\right) \\
x_{4} \\
\frac{k}{J}\left(x_{1}-x_{3}\right)
\end{array}\right] \quad g(x)=\left[\begin{array}{l}
0 \\
0 \\
0 \\
\frac{1}{J}
\end{array}\right]
$$

Therefore $n=4$ and the necessary and sufficient conditions for feedback linearization of this system are that 


$$
\operatorname{rank}\left\{g, a d_{f}(g), a d_{f}^{2}(g), a d_{f}^{3}(g)\right\}=\operatorname{rank}\left[\begin{array}{cccc}
0 & 0 & 0 & \frac{k}{I J} \\
0 & 0 & \frac{k}{I J} & 0 \\
0 & \frac{1}{J} & 0 & -\frac{k}{J^{2}} \\
\frac{1}{J} & 0 & -\frac{k}{J^{2}} & 0
\end{array}\right]=4
$$

which has rank 4 for $k>0, I, J<\infty$. Also, since vector fields $\left\{g, a d_{f}(g), a d_{f}^{2}(g)\right\}$ are constant, they form an involutive set.

$$
\left\{g, a d_{f}(g), a d_{f}^{2}(g)\right\}
$$

To see this it suffices to note that the Lie Bracket of two constant vector fields is zero. Hence the Lie Bracket of any two members of the set of vector fields in (65) is zero which is trivially a linear combination of the vector fields themselves. It follows that the system (60)- (61) is feedback linearizable. The new coordinates

$$
y_{i}=T_{i} \quad i=1, \cdots, 4
$$

are found from the conditions (67)- (68)

$$
\begin{gathered}
<d T_{1}, a d_{f}^{k}(g)>=0 \quad k=0,1, \cdots, n-2 \\
<d T_{1}, a d_{f}^{n-1}(g)>\neq 0
\end{gathered}
$$

with $n=4$, that is

$$
\begin{gathered}
<d T_{1}, g>=0 \\
<d T_{1},[f, g]>=0 \\
<d T_{1}, a d_{f}^{2}(g)>=0 \\
<d T_{1}, a d_{f}^{3}(g)>\neq 0
\end{gathered}
$$

Carrying out the above calculations leads to the system of equations

$$
\frac{\partial T_{1}}{\partial x_{2}}=0 \quad \frac{\partial T_{1}}{\partial x_{3}}=0 \quad \frac{\partial T_{1}}{\partial x_{4}}=0
$$

and

$$
\frac{\partial T_{1}}{\partial x_{1}} \neq 0
$$


From this we see that the function $T_{1}$ should be a function of $x_{1}$ alone. Therefore, we take the simplest solution

$$
y_{1}=T_{1}=x_{1}
$$

and compute

$$
\begin{gathered}
y_{2}=T_{2}=<d T_{1}, f>=x_{2} \\
y_{3}=T_{3}=<d T_{2}, f>=-\frac{M g L}{I} \sin \left(x_{1}\right)-\frac{k}{I}\left(x_{1}-x_{3}\right) \\
y_{4}=T_{4}=<d T_{3}, f>=-\frac{M g L}{I} \cos \left(x_{1}\right) \times x_{2}-\frac{k}{I}\left(x_{2}-x_{4}\right)
\end{gathered}
$$

The feedback linearizing control input $\mathrm{u}$ is found from the condition

$$
\begin{aligned}
& u=\frac{1}{\left.<d T_{4}, g\right\rangle}\left(v-<d T_{4}, f>\right) \\
& =\frac{I J}{k}(v-a(x))=\beta(x) v+\alpha(x)
\end{aligned}
$$

where

$$
\alpha(x):=\frac{M g L}{I} \sin \left(x_{1}\right)\left(x_{2}^{2}+\frac{M g L}{I} \cos \left(x_{1}\right)+\frac{k}{I}\right)+\frac{k}{I}\left(x_{1}-x_{3}\right)\left(\frac{k}{I}+\frac{k}{J}+\frac{M g L}{I} \cos \left(x_{1}\right)\right)
$$

Therefore in the coordinates $y_{1}, \cdots, y_{4}$ with the control law (79) the system becomes

$$
\begin{array}{ll}
\dot{y}_{1}=y_{2} & \dot{y}_{2}=y_{3} \\
\dot{y}_{3}=y_{4} & \dot{y}_{4}=v
\end{array}
$$

or, in matrix form,

$$
\dot{y}=A y+b v
$$

where

$$
A=\left[\begin{array}{llll}
0 & 1 & 0 & 0 \\
0 & 0 & 1 & 0 \\
0 & 0 & 0 & 1 \\
0 & 0 & 0 & 0
\end{array}\right] \quad b=\left[\begin{array}{l}
0 \\
0 \\
0 \\
1
\end{array}\right]
$$

The transformed variables $y_{1}, \cdots, y_{4}$ are themselves physically meaningful. We see that

$$
y_{1}=x_{1}=\text { link position }
$$




$$
\begin{gathered}
y_{2}=x_{2}=\text { link velocity } \\
y_{3}=\dot{y}_{2}=\text { link acceleration } \\
y_{4}=\dot{y}_{3}=\text { link jerk }
\end{gathered}
$$

Since the motion trajectory of the link is typically specified in terms of these quantities they are natural variables to use for feedback.

For given a linear system in state space form, such as (81), a state feedback control law is an input $\mathrm{v}$ of the form

$$
v=-k^{T} y+r=-\sum_{i=1}^{4} k_{i} y_{i}+r
$$

where $k_{i}$ are constants and $r$ is a reference input. If we substitute the control law (82) into (81), we obtain

$$
\dot{y}=\left(A-b k^{T}\right) y+b r
$$

Thus we see that the linear feedback control has the effect of changing the poles of the system from those determined by A to those determined by $A-b k^{T}$

When the parameters are chosen $k_{1}=62.5, k_{2}=213.6, k_{3}=204.2 k_{4}=54$, we can get step responses in Figure 4 . where k1, k2, k3 and k4 are linear feedback coefficients to place the eigenvalues of $\mathrm{A}$ in a desired location.

$$
\begin{gathered}
{\left[\begin{array}{l}
\dot{y}_{1} \\
\dot{y}_{2} \\
\dot{y}_{3} \\
\dot{y}_{4}
\end{array}\right]=\left[\begin{array}{cccc}
0 & 1 & 0 & 0 \\
0 & 0 & 1 & 0 \\
0 & 0 & 0 & 1 \\
-62.5 & -213.8 & -204.2 & -54
\end{array}\right]\left[\begin{array}{l}
y_{1} \\
y_{2} \\
y_{3} \\
y_{4}
\end{array}\right]+\left[\begin{array}{l}
0 \\
0 \\
0 \\
1
\end{array}\right] r} \\
y=\left[\begin{array}{llll}
1 & 0 & 0 & 0
\end{array}\right]\left[\begin{array}{l}
y_{1} \\
y_{2} \\
y_{3} \\
y_{4}
\end{array}\right]
\end{gathered}
$$

The internal model parameter: $K_{M}=0.016, T_{M}=3, d=8$, and the coincidence point $\mathrm{H}=10$. Response time of reference trajectory is 0.01 , and sample time is 0.01 .

For the uncertainty $\eta$, the system (38) can be written in matrix form as

$$
\dot{y}=\left(A-b k^{T}\right) y+b\{r+\eta(y, v)\}
$$

then use predictive function control strategy to reduce or overcome uncertainty of nonlinear feedback error $\eta(y, v)$, and simulation result is shown in Figure 5 for $\eta(y, v)=10 \% y_{r}$. 


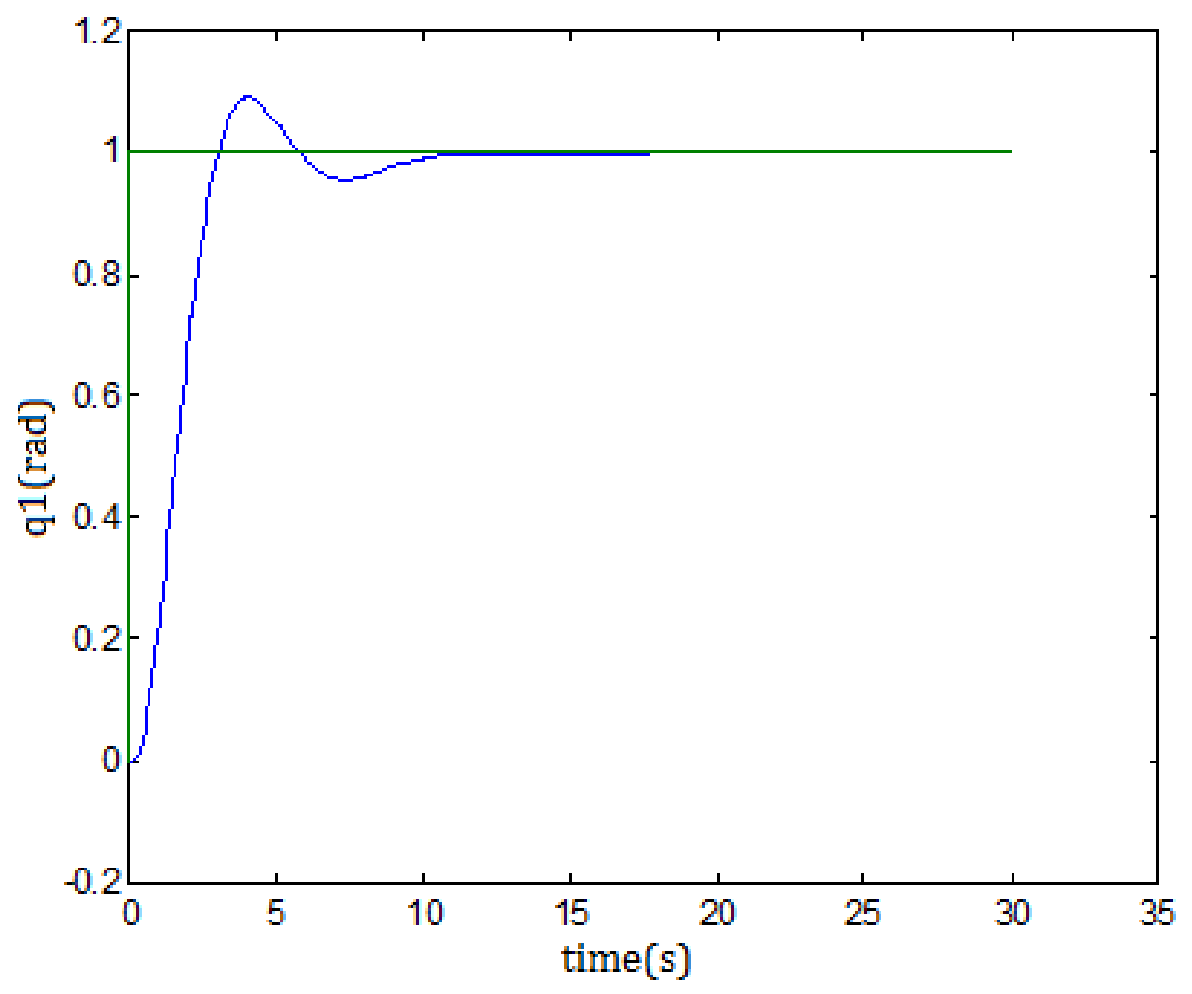

Fig. 4. link position output 


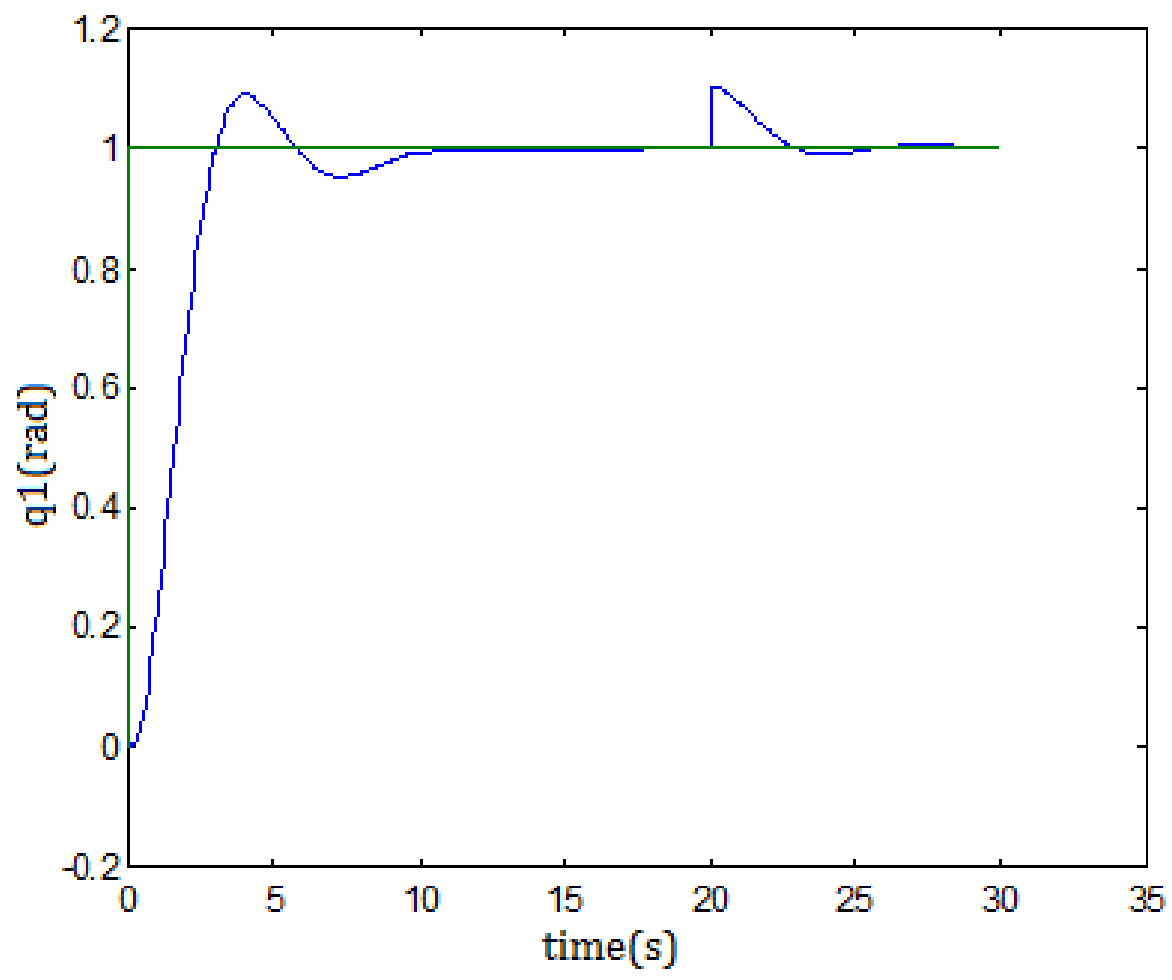

Fig. 5. link position output with uncertainty rejection 


\section{Conclusion}

A new three stage design method is presented for the single link manipulator with flexible joint. The first is feedback linearization; the second is to use pole placement to satisfy performance, and the third is to develop predictive function control to compensate uncertainty. Finally, for the same uncertainty, robustness is better than traditional method.

\section{References}

Khorasani, K. (1990). Nonlinear feedback control of flexible joint manipulators: a single link case study. IEEE Transactions on Automatic Control, Vol. 35, No. 10, pp. 1145-1149, ISSN 0018-9286

Richalet, J.; Rault, A.; Testud, J. L.; Papon, J.(1978). Model predictive heuristic control: Applications to industrial processes. Automatica, Vol. 14, No. 5, pp. 413-428, ISSN 0005-1098

Spong, M.W.; Khorasani, K. \& Kokotovic P.V. (1987). An integral manifold approach to the feedback control of flexible joint robots. IEEE Journal of Robotics and Automation, Vol. RA-3, No. 4, pp. 291-300, ISSN 0882-4967

Spong, M.W.(1987). Modeling and control of elastic joint robots. Journal of Dyn. Sys. Meas. and Cont., Vol. 109, pp.310-319, ISSN 0022-0434

Spong, M.W. (1989). On the force control problem for flexible joint manipulator. IEEE Transactions on Automatic Control, Vol. 34, No. 1, pp. 107-111, ISSN 0018-9286

E. F. Camacho, Carlos Bordons(2004). Model predictive control. Springer-Verlag London Berlin Limited. ISBN 1-85233-694-3 


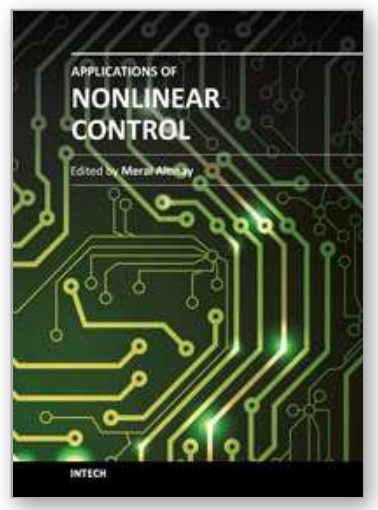

\author{
Applications of Nonlinear Control \\ Edited by Dr. Meral Altınay
}

ISBN 978-953-51-0656-2

Hard cover, 202 pages

Publisher InTech

Published online 13, June, 2012

Published in print edition June, 2012

A trend of inverstigation of Nonlinear Control Systems has been present over the last few decades. As a result the methods for its analysis and design have improved rapidly. This book includes nonlinear design topics such as Feedback Linearization, Lyapunov Based Control, Adaptive Control, Optimal Control and Robust Control. All chapters discuss different applications that are basically independent of each other. The book will provide the reader with information on modern control techniques and results which cover a very wide application area. Each chapter attempts to demonstrate how one would apply these techniques to real-world systems through both simulations and experimental settings.

\title{
How to reference
}

In order to correctly reference this scholarly work, feel free to copy and paste the following:

Zhihuan Zhang and Chao Hu (2012). Predictive Function Control of the Single-Link Manipulator with Flexible Joint, Applications of Nonlinear Control, Dr. Meral Altınay (Ed.), ISBN: 978-953-51-0656-2, InTech, Available from: http://www.intechopen.com/books/applications-of-nonlinear-control/predictive-function-control-of-thesingle-link-manipulator-with-flexible-joint

\section{INTECH}

open science | open minds

\section{InTech Europe}

University Campus STeP Ri

Slavka Krautzeka 83/A

51000 Rijeka, Croatia

Phone: +385 (51) 770447

Fax: +385 (51) 686166

www.intechopen.com

\section{InTech China}

Unit 405, Office Block, Hotel Equatorial Shanghai

No.65, Yan An Road (West), Shanghai, 200040, China

中国上海市延安西路65号上海国际贵都大饭店办公楼405单元

Phone: +86-21-62489820

Fax: +86-21-62489821 
(C) 2012 The Author(s). Licensee IntechOpen. This is an open access article distributed under the terms of the Creative Commons Attribution 3.0 License, which permits unrestricted use, distribution, and reproduction in any medium, provided the original work is properly cited. 